\title{
THE 2014 ALMA LONG BASELINE CAMPAIGN: OBSERVATIONS OF ASTEROID 3 JUNO AT 60 KILOMETER RESOLUTION*
}

\author{
Alma Partnership, T. R. Hunter ${ }^{1}$, R. Kneissl ${ }^{2,3}$, A. Moullet $^{1}$, C. L. Brogan ${ }^{1}$, E. B. Fomalont ${ }^{1,2}$, C. Vlahakis ${ }^{2,3}$, \\ Y. Asaki ${ }^{4,5}$, D. Barkats ${ }^{2,3}$, W. R. F. Dent ${ }^{2,3}$, R. E. Hills ${ }^{6}$, A. Hirota ${ }^{2,4}$, J. A. Hodge ${ }^{1}$, C. M. V. ImPellizzeri ${ }^{1,2}$, E. LiUzzo ${ }^{7}$,

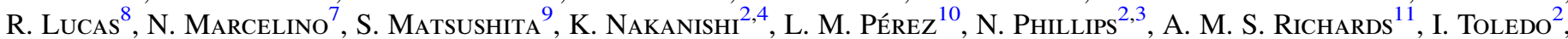 \\ R. Aladro ${ }^{3}$, D. Broguiere ${ }^{12}$, J. R. Cortes ${ }^{1,2}$, P. C. Cortes ${ }^{1,2}$, D. Espada ${ }^{2,4}$, F. Galarza ${ }^{2}$, D. Garcia-Appadoo ${ }^{2,3}$, \\ L. Guzman-Ramirez ${ }^{3}$, A. S. Hales ${ }^{1,2}$, E. M. Humphreys ${ }^{13}$, T. Jung ${ }^{14}$, S. KAmeno ${ }^{2,4}$, R. A. Laing ${ }^{13}$, S. LeON ${ }^{2,3}$, G. MARCONI ${ }^{2,3}$, \\ A. Mignano ${ }^{7}$, B. Nikolic ${ }^{6}$, L.-A. Nyman ${ }^{2,3}$, M. RadiszCZ ${ }^{2}$, A. Remijan ${ }^{1,2}$, J. A. Rodón ${ }^{3}$, T. SAWADA ${ }^{2,4}$, S. TAKAHASHi ${ }^{2,4}$, \\ R. P. J. Tilanus ${ }^{15}$, B. Vila Vilaro ${ }^{2,3}$, L. C. Watson $^{3}$, T. Wiklind ${ }^{2,3}$, I. De Gregorio-Monsalvo ${ }^{2,3}$, J. Di Francesco ${ }^{16}$, \\ J. Mangum ${ }^{1}$, H. Francke ${ }^{2}$, J. Gallardo ${ }^{2}$, J. Garcia ${ }^{2}$, S. Gonzalez ${ }^{2}$, T. Hill ${ }^{2,3}$, T. Kaminski ${ }^{3}$, Y. Kurono ${ }^{2,4}$, C. Lopez ${ }^{2}$, \\ F. Morales ${ }^{2}$, K. Plarre ${ }^{2}$, S. Randall ${ }^{13}$, T. VAn Kempen ${ }^{15}$, L. Videla ${ }^{2}$, E. Villard ${ }^{2,3}$, P. Andreani ${ }^{13}$, \\ J. E. HibBARD ${ }^{1}$, AND K. TATEMATSU ${ }^{4}$ \\ ${ }^{1}$ National Radio Astronomy Observatory, 520 Edgemont Road, Charlottesville, VA 22903, USA; thunter@nrao.edu \\ ${ }^{2}$ Joint ALMA Observatory, Alonso de Córdova 3107, Vitacura, Santiago, Chile \\ ${ }^{3}$ European Southern Observatory, Alonso de Córdova 3107, Vitacura, Santiago, Chile \\ ${ }^{4}$ National Astronomical Observatory of Japan, 2-21-1 Osawa, Mitaka, Tokyo 181-8588, Japan \\ ${ }^{5}$ Institute of Space and Astronautical Science (ISAS), Japan Aerospace Exploration Agency (JAXA), \\ 3-1-1 Yoshinodai, Chuo-ku, Sagamihara, Kanagawa 252-5210 Japan \\ ${ }^{6}$ Astrophysics Group, Cavendish Laboratory, J.J. Thomson Avenue, Cambridge CB3 0HE, UK \\ 7 INAF, Istituto di Radioastronomia, Via P. Gobetti 101, I-40129, Bologna, Italy \\ ${ }^{8}$ Institut de Planétologie et d'Astrophysique de Grenoble (UMR 5274), BP 53, F-38041 Grenoble Cedex 9, France \\ ${ }^{9}$ Institute of Astronomy and Astrophysics, Academia Sinica, P.O. Box 23-141, Taipei 106, Taiwan \\ ${ }^{10}$ National Radio Astronomy Observatory, P.O. Box O, Socorro, NM 87801, USA \\ ${ }^{11}$ Jodrell Bank Centre for Astrophysics, School of Physics and Astronomy, University of Manchester, Oxford Road, Manchester M13 9PL, UK \\ ${ }^{12}$ Institut de Radioastronomie Millimétrique (IRAM), 300 rue de la Piscine, Domaine Universitaire, F-38406 Saint Martin d'Hères, France \\ ${ }^{13}$ European Southern Observatory, Karl-Schwarzschild-Str. 2, D-85748 Garching bei München, Germany \\ ${ }^{14}$ Korea Astronomy and Space Science Institute, Daedeokdae-ro 776, Yuseong-gu, Daejeon 305-349, Korea \\ ${ }^{15}$ Leiden Observatory, Leiden University, P.O. Box 9513, 2300 RA Leiden, The Netherlands \\ ${ }^{16}$ National Research Council Herzberg Astronomy \& Astrophysics, 5071 West Saanich Road, Victoria, BC V9E 2E7, Canada \\ Received 2015 February 25; accepted 2015 March 13; published 2015 July 14
}

\begin{abstract}
We present Atacama Large Millimeter/submillimeter Array (ALMA) $1.3 \mathrm{~mm}$ continuum images of the asteroid 3 Juno obtained with an angular resolution of $0 . " 042(60 \mathrm{~km}$ at $1.97 \mathrm{AU})$. The data were obtained over a single $4.4 \mathrm{hr}$ interval, which covers $60 \%$ of the $7.2 \mathrm{hr}$ rotation period, approximately centered on local transit. A sequence of 10 consecutive images reveals continuous changes in the asteroid's profile and apparent shape, in good agreement with the sky projection of the three-dimensional model of the Database of Asteroid Models from Inversion Techniques. We measure a geometric mean diameter of $259 \pm 4 \mathrm{~km}$, in good agreement with past estimates from a variety of techniques and wavelengths. Due to the viewing angle and inclination of the rotational pole, the southern hemisphere dominates all of the images. The median peak brightness temperature is $215 \pm 13 \mathrm{~K}$, while the median over the whole surface is $197 \pm 15 \mathrm{~K}$. With the unprecedented resolution of ALMA, we find that the brightness temperature varies across the surface with higher values correlated to the subsolar point and afternoon areas and lower values beyond the evening terminator. The dominance of the subsolar point is accentuated in the final four images, suggesting a reduction in the thermal inertia of the regolith at the corresponding longitudes, which are possibly correlated to the location of the putative large impact crater. These results demonstrate ALMA's potential to resolve thermal emission from the surface of main belt asteroids and to measure accurately their position, geometric shape, rotational period, and soil characteristics.
\end{abstract}

Key words: minor planets, asteroids: general - minor planets, asteroids: individual (3 Juno) - planets and satellites: surfaces - techniques: interferometric

\section{INTRODUCTION}

Discovered in 1804, Juno was the third main-belt asteroid identified, following Ceres and Pallas. The first reasonably accurate measurement of Juno's diameter was performed with filar micrometers on the Great Lick Refractor (Barnard 1895) and the Yerkes 40 inch Refractor (Barnard 1900), yielding a

\footnotetext{
* This article is part of the ALMA Long Baseline Campaign collection. An introduction and the full list of articles can be found at http://iopscience.iop. org/2041-8205/page/Focus_on_the_ALMA_Long_Baseline_Campaign.
}

value of $193 \pm 20 \mathrm{~km}$ (see also Dollfus 1971). A modern measurement of its physical cross section came from 15 station stellar occultation data, yielding a mean diameter of $267 \pm 5 \mathrm{~km}$ with a significant ellipticity (Millis et al. 1981). Optical speckle interferometry soon produced a size measurement consistent with the occultation result (Baier \& Weigelt 1983). Like most asteroids, Juno's light-curve is double-peaked with two maxima and two minima (e.g., Birch \& Taylor 1989), indicative of a non-spherical shape. Based on light-curve inversion, Juno has a unique rotational pole that is significantly 
tilted with respect to the ecliptic (Magnusson 1986; Dotto et al. 1995), and its period of $7.209531 \mathrm{hr}$ is known to high accuracy (Kaasalainen et al. 2002). This information, combined with recent, near-infrared adaptive optics (AO) imaging led to a triaxial ellipsoid model with axis lengths of 297, 233, and $222 \mathrm{~km}$ (Drummond \& Christou 2008). A three-dimensional model with 2036 faces and 1020 vertices based on a combination of the historical optical light curves and two occultations (Kaasalainen et al. 2002; Durech et al. 2011) is hosted by the Database of Asteroid Models from Inversion Techniques (DAMIT; Ďrech et al. 2010).

Juno is a member of the S-class of asteroids (Chapman et al. 1975), which have a stony composition of iron-bearing silicates and metallic iron as inferred primarily from their $1 \mu \mathrm{m}$ spectral absorption feature (e.g., Gaffey et al. 1993a and references therein). The optical Small Main-Belt Asteroid Spectroscopic Survey (SMASSII) assigns it subclass Sk as a transition object toward the K-class, which exhibits a shallower $1 \mu \mathrm{m}$ feature (Bus \& Binzel 2002). Infrared Space Observatory spectra of Juno show an $8-11.5 \mu \mathrm{m}$ feature that is consistent with the laboratory measurements of the silicate minerals pyroxine and olivine (Dotto et al. 2000). Evidence for surface features on Juno have been suggested by the variation as a function of rotation angle of its optical colors (Degewij et al. 1979; Schroll et al. 1981) and linear polarization (Shinokawa et al. 2002; Takahashi et al. 2009), and by a sequence of optical AO images, which suggested a large impact crater (Baliunas et al. 2003). Somewhat surprisingly, there are no published images of Juno from the Hubble Space Telescope (Dotto et al. 2002), and there have been no spacecraft encounters as of yet.

As a powerful new tool in the study of solar system bodies, the Atacama Large Millimeter/submillimeter Array (ALMA; Hills et al. 2010) will be able to map the shape and surface temperature distributions of hundreds of main belt asteroids and Jupiter Trojans (Lovell 2008; Busch 2009). The reason is twofold. First, the absorption length of (sub)millimeter photons (Campbell \& Ulrichs 1969) on asteroid surfaces is comparable to the thermal skin depth of the diurnal wave (typically a few to 10 wavelengths; Spencer \& Lebofsky 1989); thus, these wavelengths are well matched to probe the thermal response of this material and should provide information on the thickness, structure, and nature of the regolith (Lagerros 1996; Chamberlain et al. 2007). Indeed, the recent flybys of the main belt asteroids 21 Lutetia and 2867 Šteins by the Rosetta mission have yielded important measurements of the thermal inertia and emissivity of their surface material (Section 4) using its (sub) millimeter radiometers (Gulkis et al. 2010, 2012). Second, ALMA has exquisite continuum brightness temperature sensitivity at small angular scales. For example, the 50 antenna, full-bandwidth sensitivity in one minute at $300 \mathrm{GHz}(1 \mathrm{~mm})$ at the highest angular resolution (13 mas) is $10 \mathrm{~K}$. Since their physical temperatures are typically 100-200 K, ALMA can effectively image these bodies down to linear resolutions of $10 \mathrm{~km}$ (at a distance $\Delta=1 \mathrm{AU}$ ) with high signal-to-noise ratio $(\mathrm{S} / \mathrm{N})$, enabling the use of the powerful tool of self-calibration. As a significant step toward demonstrating this capability, in this Letter we present the first millimeter wavelength images to resolve the surface of Juno, which were obtained during the recent campaign to commission ALMA's long baseline capabilities (ALMA Partnership et al. 2015, hereafter ALMA I). The availability of the DAMIT model provides an excellent test of ALMA's imaging performance, while the resulting images provide new details on the surface conditions of Juno.

\section{OBSERVATIONS}

An approximately 53 minute length scheduling block (SB) to observe the $1.3 \mathrm{~mm}(233 \mathrm{GHz})$ continuum emission from Juno was executed five consecutive times on 2014 October 19 starting at 09:15 UT (43 minutes before local sunrise) and ending at 13:38 UT. Four spectral windows were used, each with $2 \mathrm{GHz}$ bandwidth, 128 channels, and dual polarization. Center frequencies were 224, 226, 240, and $242 \mathrm{GHz}$. All necessary calibration observations were performed in each execution of the SB. An additional focus measurement and adjustment were performed prior to the fifth execution (two hours after sunrise) as per normal operations. The SB included an external ephemeris with 4 minute sampling obtained from Jet Propulsion Lab (JPL) Horizons, ${ }^{17}$ which reports a $3 \sigma$ uncertainty of 60 mas in right ascension and 26 mas in declination.

Calibration and imaging was performed in $\mathrm{CASA}^{18}$ version 4.2.2. The complex gain calibration cycle time was $68 \mathrm{~s}$, with Juno being observed for $48 \mathrm{~s}$ and the gain calibrator (J0757 +0956 ) for $15 \mathrm{~s} \mathrm{(5:7} \mathrm{away).} \mathrm{Data} \mathrm{from} 27$ to 31 antennas were used, ranging in projected baseline length from 0.02 to $10 \mathrm{M} \lambda$ $(26 \mathrm{~m}$ to $13 \mathrm{~km})$. The zenith precipitable water vapor varied from 1.4 to $1.6 \mathrm{~mm}$. Bandpass and flux calibration are based on observations of the quasar J0750+1231 in each SB. This quasar is an ALMA calibration grid source (van Kempen et al. 2014) for which a linear interpolation in frequency from the measurements nearest in time at $3 \mathrm{~mm}$ (7 days) and $0.87 \mathrm{~mm}$ (18 days) yields an assumed flux density of $0.64 \pm 0.04 \mathrm{Jy}$ at $233 \mathrm{GHz}$ with a spectral index of -0.66 . The calibrator flux density measurements were stable over many weeks, and we estimate our flux scale to be accurate to 6\%. The mean flux density derived for the gain calibrator was quite consistent across the five executions, differing by a maximum of $2.4 \%$ from the weighted mean of all executions $(0.5916 \pm$ $0.0007 \mathrm{Jy})$. Thus, there was very little decorrelation on timescales shorter than the integration time $(1.92 \mathrm{~s})$. As shown in Table 1, the span of observations was approximately centered on the time of transit of Juno.

Following calibration, the $u v$ data from each execution were split into two halves, with the duration and time on source of each half being $\sim 18$ and $\sim 10$ minutes, respectively. The 10 resulting data sets (epochs) were imaged individually using a robust weighting parameter of zero. Phase-only self-calibration was then performed, initially with a time interval of $300 \mathrm{~s}$, followed by a refinement with a time interval of $15 \mathrm{~s}$. The final execution showed phase-cal solutions of somewhat larger magnitude and higher variability than the rest, with a loss of some antennas on the outermost pads. Amplitude selfcalibration on a timescale of $300 \mathrm{~s}$ was then performed. The final images were constructed using multi-scale clean (Rau \& Cornwell 2011) with deconvolution scales of 0,5 , and 15 pixels to avoid image artifacts caused by the clean instability that can occur when modeling an extended source using only delta functions. The image dynamic range after self-calibration was 120 . This improvement factor of two to six indicates that a

\footnotetext{
17 http://ssd.jpl.nasa.gov/horizons.cgi

18 http://casa.nrao.edu
} 
Table 1

Parameters of the $1.3 \mathrm{~mm}$ ALMA Images of Juno

\begin{tabular}{lccccc}
\hline \hline $\begin{array}{l}\text { Image } \\
\text { No. }\end{array}$ & Epoch & Elapsed Time & Elevation & \multicolumn{2}{c}{$\begin{array}{c}\text { Rotation } \\
\text { Phase }\end{array}$} \\
\cline { 5 - 7 } & $($ MJD $)$ & $($ minutes $)$ & $\left({ }^{\circ}\right)$ & $\phi_{1}{ }^{\text {a }}$ & $\phi_{2}{ }^{\text {b }}$ \\
\hline 0 & 56949.39167 & 0 & 53.2 & 0.33 & 0.18 \\
1 & 56949.40417 & 18 & 55.7 & 0.37 & 0.22 \\
2 & 56949.42917 & 36 & 59.5 & 0.45 & 0.30 \\
3 & 56949.44167 & 73 & 60.5 & 0.49 & 0.35 \\
4 & 56949.46667 & 110 & 60.5 & 0.58 & 0.43 \\
5 & 56949.48056 & 128 & 59.4 & 0.62 & 0.47 \\
6 & 56949.51111 & 173 & 54.7 & 0.72 & 0.58 \\
7 & 56949.52500 & 192 & 51.9 & 0.77 & 0.62 \\
8 & 56949.54861 & 226 & 46.1 & 0.85 & 0.70 \\
9 & 56949.56111 & 244 & 42.6 & 0.89 & 0.74 \\
\hline
\end{tabular}

${ }^{\text {a }}$ Phase with respect to $\psi_{0}$ of the Drummond \& Christou (2008) triaxial model using the rotation period of 0.300397125 days (Kaasalainen et al. 2002). In the 8.5 year interval, the phase accumulated uncertainty is \pm 0.01 .

${ }^{\mathrm{b}}$ Phase with respect to AO observations of Baliunas et al. (2003; zero point taken to be 50371.24167) using the same rotation period. In the 18 year interval, the accumulated phase uncertainty is \pm 0.02 .

significant residual phase error was present after normal calibration. Thus, it is important to realize that any usage of calibrated visibilities for direct modeling (e.g., Viikinkoski et al. 2015) must either apply the imaging self-calibration solutions or include antenna-based phase solutions as a model parameter to be solved (Hezaveh et al. 2013). As expected when the initial self-calibration model has high $\mathrm{S} / \mathrm{N}$, the intensity-weighted centroid of the source before and after selfcalibration is consistent to within a fraction of the (5 mas) pixel size $(<0.2$ in most images, and $<0.3$ in the final two images). Because we used an accurate VLBI position for the gain calibrator (07:57:06.64296, +09:56:34.8525; Lanyi et al. 2010), we expect our images to follow ALMA's measured astrometric performance ( 3 mas; ALMA I). ${ }^{19}$ The images vary in the size of the synthesized beam from $31.8 \times 23.7$ mas to $41.8 \times 36.1$ mas, with a mean position angle of $30^{\circ} \pm 11^{\circ}$. These images are publicly available from the ALMA Science Verification page. ${ }^{20}$ To present matched resolution images, we smoothed the images with a two-dimensional elliptical Gaussian to obtain a circular beam of 42.0 mas, which at the Earth-Juno distance of $\Delta=1.97$ AU corresponds to $60 \mathrm{~km}$. No near-field correction was applied to the $u v$ data, but Juno was beyond the Fraunhofer distance for the longest baseline, and the self-calibration process may mitigate the residual effects.

\section{RESULTS}

\subsection{Viewing Geometry}

Juno's orbit has a mean radius of $2.67 \mathrm{AU}$ with significant eccentricity $(0.25)$ and inclination $\left(13^{\circ}\right)$. As shown in Figure 1, the true anomaly was $37^{\circ} .5$ during our observations, with a

\footnotetext{
19 The ALMA control system does not account for the finite distance of solar system targets when computing the gravitational deflection of light by the Sun to apply to the astrometric ephemeris, which causes the phase transfer from the quasar to introduce a systematic position error of $\approx 1.4$ mas for these observations of Juno according to the formulas of Cowling (1984).

${ }^{20} \mathrm{http} / / /$ almascience.org/alma-data/science-verification. Additional details including the calibration and imaging scripts are available from http://casaguides.nrao.edu/index.php?title=ALMA2014_LBC_SVDATA.
}

solar phase angle of $-28^{\circ}$ yielding an illumination percentage of $94 \%$. We have plotted the orientation of the rotational pole inferred from parametric blind deconvolution of near-infrared AO images (ecliptic longitude $\lambda=118^{\circ}$ and latitude $\beta=+30^{\circ}$ ), which has an uncertainty of $13^{\circ}$ (Drummond \& Christou 2008). The direction of Juno was $\lambda=124^{\circ} .7, \beta=-13.2$, thus our viewing angle was $48^{\circ}$ from the polar axis but nearly coplanar, with the southern hemisphere dominating the view, as shown in the inset of Figure 1. The angular smearing due to the $15^{\circ}$ of axial rotation during each 18 minute image leads to 21 mas of linear smearing at the mean radius, which is half the beam size, leading to $<12 \%$ loss in resolution. The mean epochs of the images are listed in Table 1, along with the corresponding rotational phases computed with respect to the zero time point of the Drummond \& Christou (2008) triaxial model and with respect to the optical AO observations of Baliunas et al. (2003).

\subsection{Images}

The 10 images of Juno are shown in Figure 2. The absolute position was measured in each image by computing the centroid of all pixels above the $5 \sigma$ level, where $\sigma$, the image rms, was defined by an annulus surrounding the object (see Table 2 notes). These pixels were weighted uniformly to avoid influence of surface brightness variations across the face of the object. The difference between the centroid position and the image phase center yields the observed offset from the JPL ephemeris, which is stable in right ascension at $\approx+60$ mas, but slowly varying in declination. The integrated flux density of the source was measured by integrating over all pixels above the $3 \sigma$ level. The peak positions, intensities, and the corresponding Planck brightness temperatures $\left(T_{B}\right)$ are also listed in Table 2. Note that these $T_{B}$ differ from the Rayleigh-Jeans approximation by $+5.6 \mathrm{~K}$. An estimate of the median brightness temperature across the surface was computed for each image by finding the median pixel intensity in the clean component model image and dividing by the solid angle of a pixel. The result is typically $10-15 \mathrm{~K}$ below the peak $T_{B}$.

\subsection{Size and Shape Measurement}

In order to obtain a size measurement independent of the existing shape models, we consider the underlying source to be an elliptical disk with uniform brightness. First, we fit the observed image from each epoch with a two-dimensional elliptical Gaussian, recording the position angle and major and minor axes as the target parameters. We then create a disk model image using the target parameters, but with the major and minor axes increased by $30 \%$ to account for the bulk of the size underestimate resulting from the Gaussian brightness profile. We then convolve this disk model image with a 42 mas beam to match the observations. Next, we iteratively refined the disk model parameters until an elliptical Gaussian fit to the convolved disk model image matched the target parameters. The resulting uniform brightness elliptical disk model parameters for each epoch are listed in Table 2. The geometric mean of the median of the major and minor axes of the 10 elliptical disk models $\left(0.181 \pm 0.0^{\prime \prime} 003\right)$ corresponds to $d_{\mathrm{mm}}=259 \pm 4 \mathrm{~km}$. This mean diameter is consistent with the size derived from $250 \mathrm{GHz}$ single-dish flux density measurements under the assumption of unity emissivity $\left(\epsilon_{\nu}=1 ; d_{\mathrm{mm}}=253.4 \pm 7.4\right.$; Altenhoff et al. 1994). The mean 


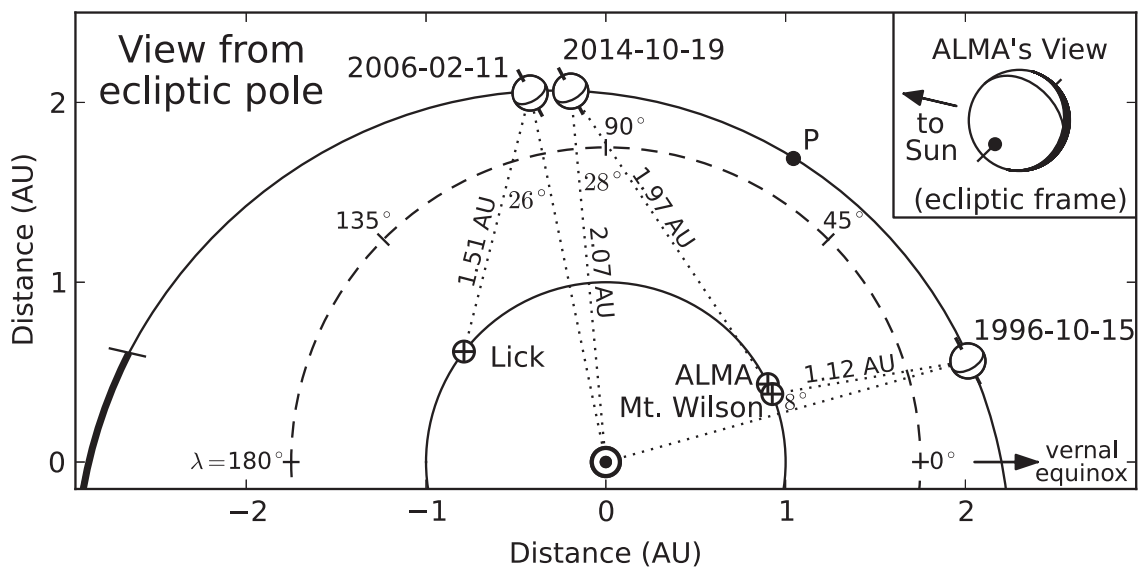

Figure 1. As viewed from the ecliptic pole, this diagram shows the alignment of Earth and Juno in their respective orbits on the three dates of observational data discussed: ALMA (this paper), Mt. Wilson Observatory (Baliunas et al. 2003), and Lick Observatory (Drummond \& Christou 2008). The scale of heliocentric ecliptic coordinates $(\lambda, \beta)$ is indicated by the dashed line circle. The three angles inside the dotted lines correspond to the Sun-target-Earth angle that describes the solar illumination phase. The nominal rotational pole (toward $\lambda=118^{\circ}, \beta=+30^{\circ}$; Drummond \& Christou 2008) and its corresponding equator are drawn onto a spherical representation of Juno for reference. Juno's perihelion at $\lambda=58.3$ is marked by the dot labeled "P." The portion of the orbit above the ecliptic is shown by the thick line, which begins at the ascending node $\left(\lambda=+169^{\circ} .9\right)$. Inset: the point of view of the ALMA observations (in the ecliptic coordinate frame), which is dominated by the southern hemisphere. Juno's south pole and equator are marked, as is the evening terminator and unlit side that reflects Juno's solar phase angle and heliocentric latitude $\left(\beta=-13^{\circ} .2\right.$ viewed from Earth) at the time of observation.
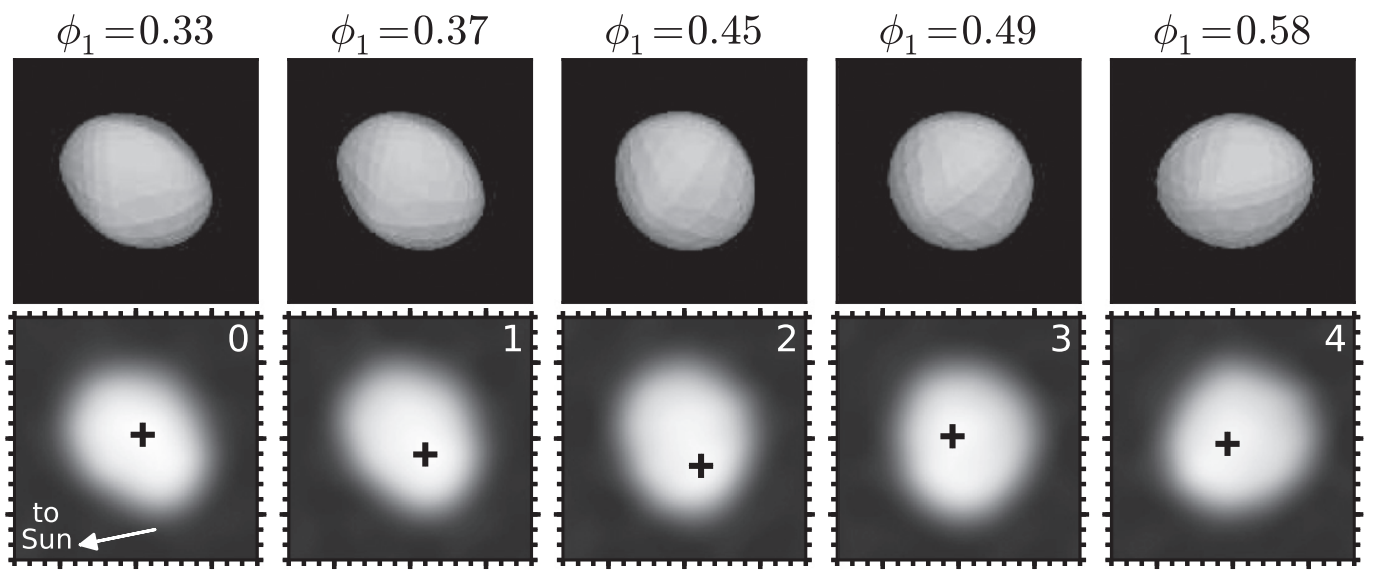

$$
\phi_{1}=0.62
$$
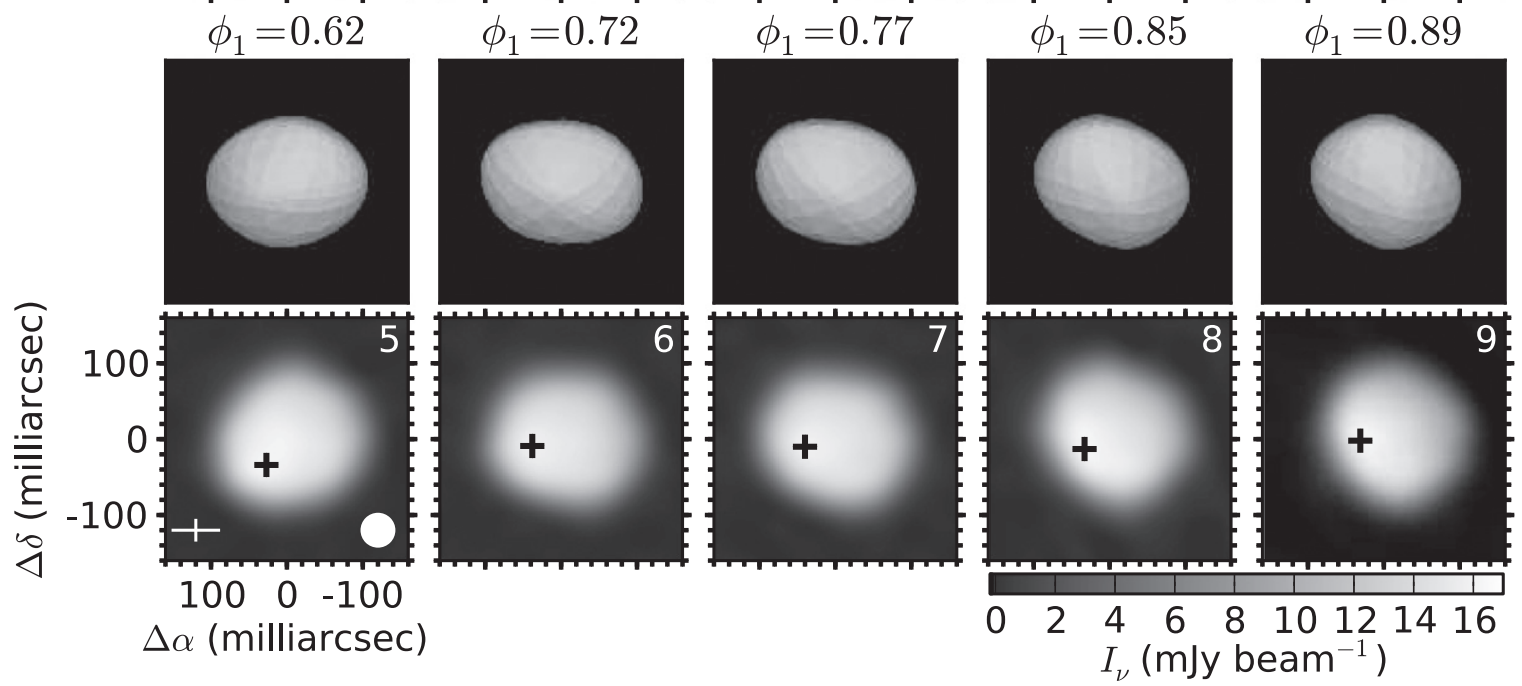

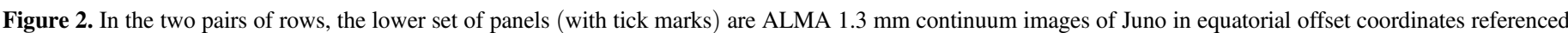

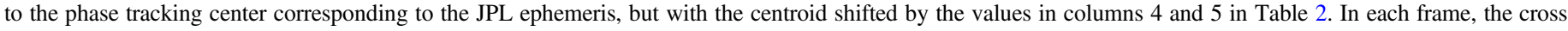

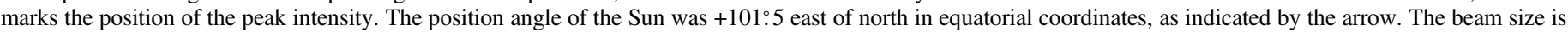

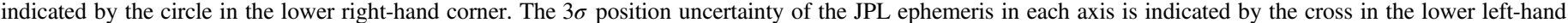

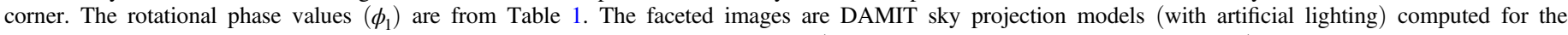
corresponding mean epoch of each image and are shown on the same angular scale (the models have a $12 \%$ uncertainty in scale). 
Derived Properties from the $1.3 \mathrm{~mm}$ ALMA Observations

\begin{tabular}{|c|c|c|c|c|c|c|c|c|c|}
\hline \multirow{3}{*}{$\begin{array}{l}\text { Image } \\
\text { No. }\end{array}$} & \multirow{2}{*}{\multicolumn{2}{|c|}{$\begin{array}{l}\text { Centroid of Juno } \\
\text { Absolute Position }\end{array}$}} & \multirow{3}{*}{$\begin{array}{c}\text { Offset }^{\mathrm{b}} \\
\Delta \alpha, \Delta \delta \\
(\mathrm{mas}, \mathrm{mas})\end{array}$} & \multirow{3}{*}{$\begin{array}{c}\text { Integrated } \\
\text { Flux Density }{ }^{\mathrm{c}, \mathrm{d}} \\
(\mathrm{mJy})\end{array}$} & \multirow{3}{*}{$\begin{array}{c}\text { Peak } \\
\text { Intensity }{ }^{\mathrm{d}, \mathrm{e}} \\
\left(\mathrm{mJy}^{-1} \text { beam }^{-1}\right)\end{array}$} & \multicolumn{2}{|c|}{$T_{B}$} & \multirow{3}{*}{$\begin{array}{c}T_{B} \text { Peak } \\
\text { Position } \\
\text { (mas, mas) }\end{array}$} & \multirow{3}{*}{$\begin{array}{c}\text { Elliptical Disk Model } \\
\text { Parameters }^{\mathrm{g}} \\
\left(\operatorname{mas} \times \operatorname{mas}\left(^{\circ}\right)\right)\end{array}$} \\
\hline & & & & & & \multirow{2}{*}{$\begin{array}{c}\operatorname{Peak}^{\mathrm{d}} \\
(\mathrm{K})\end{array}$} & \multirow{2}{*}{$\begin{array}{l}\text { Median }^{\mathrm{d}, \mathrm{f}} \\
(\mathrm{K})\end{array}$} & & \\
\hline & $\alpha(\mathbf{J} 2000)$ & $\delta(\mathbf{J} 2000)$ & & & & & & & \\
\hline 0 & 081446.8474 & +061908.554 & $+59,-5$ & $199.7 \pm 1.5$ & $17.0 \pm 0.09$ & $222 \pm 1$ & 213 & $-9,-5$ & $200 \times 157(+47 \pm 3)$ \\
\hline 1 & 081447.8979 & +06 1902.586 & $+55,+4$ & $198.1 \pm 1.5$ & $16.8 \pm 0.09$ & $220 \pm 1$ & 208 & $-20,+21$ & $199 \times 159(+37 \pm 3)$ \\
\hline 2 & 081449.9975 & +06 1850.632 & $+52,+14$ & $199.2 \pm 1.5$ & $16.5 \pm 0.09$ & $216 \pm 1$ & 202 & $-22,+36$ & $196 \times 165(+16 \pm 4)$ \\
\hline 3 & 081451.0474 & +06 1844.645 & $+62,+13$ & $200.6 \pm 1.5$ & $16.6 \pm 0.09$ & $218 \pm 1$ & 200 & $+8,-3$ & $192 \times 169(+0 \pm 6)$ \\
\hline 4 & 081453.1449 & +06 1832.674 & $+63,+23$ & $202.9 \pm 1.5$ & $16.3 \pm 0.09$ & $214 \pm 1$ & 197 & $+7,+7$ & $191 \times 175(-47 \pm 8)$ \\
\hline 5 & 081454.3100 & +06 1826.017 & $+63,+26$ & $201.8 \pm 1.4$ & $16.2 \pm 0.09$ & $213 \pm 1$ & 196 & $+27,+34$ & $194 \times 173(-67 \pm 6)$ \\
\hline 6 & 081456.8740 & +061811.357 & $+62,+31$ & $195.8 \pm 1.5$ & $15.7 \pm 0.09$ & $207 \pm 1$ & 186 & $+38,+9$ & $200 \times 170(-99 \pm 4)$ \\
\hline 7 & 081458.0396 & +061804.685 & $+55,+30$ & $196.1 \pm 1.5$ & $15.8 \pm 0.09$ & $208 \pm 1$ & 188 & $+40,+10$ & $200 \times 169(-108 \pm 4)$ \\
\hline 8 & 081500.0242 & +061753.355 & $+62,+47$ & $188.4 \pm 1.8$ & $16.3 \pm 0.11$ & $215 \pm 2$ & 187 & $+33,+13$ & $192 \times 167(-126 \pm 5)$ \\
\hline 9 & 081501.0759 & +06 1747.339 & $+64,+43$ & $181.6 \pm 2.0$ & $16.4 \pm 0.12$ & $215 \pm 2$ & 179 & $+31,+2$ & $187 \times 168(-137 \pm 6)$ \\
\hline Median $^{\text {h }}$ & $\ldots$ & $\ldots$ & $+60.5,+24.5$ & $198.7 \pm 2.7$ & $16.4 \pm 0.2$ & $215 \pm 3$ & $197 \pm 9$ & $\ldots$ & $194 \times 169$ \\
\hline
\end{tabular}

${ }^{\text {a }}$ See section Section 3.2 for details of the centroid calculation. The systematic uncertainty is estimated to be 3 mas. All coordinates are in the ICRF of the ICRS (Epoch J2000.0).

${ }^{\mathrm{b}}$ Offset of centroid with respect to the phase center of the image, which follows the JPL Horizons ephemeris.

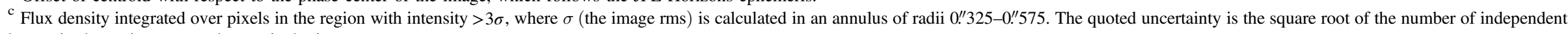
beams in the region $\times 3 \sigma$, where $\sigma$ is the image rms.

d The systematic flux calibration uncertainty of $6 \%$ is not included.

${ }^{\mathrm{e}}$ The quoted uncertainty is the image rms.

${ }^{\mathrm{f}}$ The median brightness temperature $\left(T_{B}\right)$ is computed using the Planck equation with the median pixel intensity in the clean model (within the central $\left.0 .{ }^{\prime \prime} 15\right)$ and the solid angle per pixel.

$\mathrm{g}$ Major axis, minor axes, and position angle east of north. The fit uncertainty on the major and minor axes is 3 mas.

${ }^{\mathrm{h}}$ Where listed, the uncertainty is the median absolute deviation from the median. 
diameter is also in reasonable agreement with the triaxial geometric mean $(250 \pm 5 \mathrm{~km})$ of the three axes of the Drummond \& Christou (2008) triaxial ellipsoid model, the equivalent diameter of the Durech et al. (2011) model $(252 \pm 29 \mathrm{~km})$, and the effective diameter measured by radar $(265 \pm 30 \mathrm{~km}$; Magri et al. 2007). The IRAS Minor Planet Survey (IMPS) inferred a radiometric mean diameter of $234 \pm 11 \mathrm{~km}$, but IMPS diameters are systematically low compared to occultation diameters (Tedesco et al. 2004). We note that our assumption of uniform brightness is somewhat unphysical, as the daytime surface temperature will vary as a function of local hour and latitude in more realistic thermal models (Lebofsky \& Spencer 1989). We estimate that our method will yield sizes that are $\approx 2 \%-4 \%$ smaller than the mean physical size. Thus, further detailed comparisons of the millimeter images to any specific shape model should be performed in the context of a thermal model.

\subsection{Comparison to DAMIT Shape Model}

The model images shown in Figure 2 were obtained using the DAMIT online tool ${ }^{21}$ (see also the Interactive Service for Asteroid Models ${ }^{22}$; Marciniak et al. 2012). This tool provides a prediction of the projected appearance of the asteroid on the sky as viewed from Earth in equatorial coordinate orientation at any observed Julian date. The light travel time effect is taken into account (in this case, 16.4 minutes). The DAMIT prediction for the ESO $1.5 \mathrm{~m}$ speckle observation of Baier \& Weigelt (1983) is in excellent agreement with the shape of the observed image, obtained over 34 years ago. Likewise, the shape of the DAMIT prediction is in good agreement with most of the ALMA images. The images we show here use artificial lighting in order to show the full geometrical extent of the body, which will emit millimeter emission that is only mildly modulated by solar illumination. In a qualitative sense, it is perhaps images 4 and 5 that are most discrepant from the model in terms of ellipticity and orientation. As for angular scale, the quoted accuracy of the DAMIT images is $\pm 12 \%$. The ALMAderived major axes (Table 2 ) are systematically $\approx 6 \%$ smaller than the maximum extent in the DAMIT images; however, much of this difference could be due to the simplistic model used in Section 3.3.

\subsection{Surface Features}

To accentuate the variation of brightness across the object, we have created a model image of uniform brightness corresponding to each of the 10 images. We begin with the clean component model image and compute the median value within the central 0"15 diameter (Section 3.2). We then place this value into all pixels of the clean model that are inside the $40 \%$ level in the clean image and place zero elsewhere. This approach leads to a model image comparable to the angular dimensions of Juno. We then convolve this model with the 42 mas beam and subtract it from the clean image. This subtraction enables the identification of areas of lower versus higher brightness temperature, regardless of the relative calibration accuracy between the different images. The results are shown in Figure 3. In most of the frames there is a consistent pattern of the northwest edge being the coolest portion, which matches the location of the evening terminator.

\footnotetext{
21 http://astro.troja.mff.cuni.cz/projects/asteroids3D/web.php

22 http://isam.astro.amu.edu.pl
}

Also, the warmest part of the image appears correlated with the subsolar point. In the first five images, the afternoon area following the subsolar point is the warmest point. Meanwhile, in the last four images, the warmest point is at (or very close to) the subsolar point. The temperature contrast becomes particularly pronounced in the final two images as the surface median $T_{B}$ declines (Table 2).

\section{DISCUSSION}

In the simple equilibrium model, the expected disk-averaged brightness temperature, $T_{B}(\nu)$, of an asteroid is determined by its spectral emissivity, $\epsilon_{\nu}$, and its mean equilibrium temperature, $T_{\text {eq }}$ :

$$
\begin{gathered}
T_{B}(\nu)=\epsilon_{\nu} T_{\text {eq }} \\
T_{\text {eq }}=f(1-A)^{1 / 4} r^{-1 / 2},
\end{gathered}
$$

where $A$ is its bolometric Bond albedo, $r$ is its heliocentric distance in $\mathrm{AU}$, and $f=329 \mathrm{~K}$ for non-rotating objects and $277 \mathrm{~K}$ for fast-rotating objects (Kellermann 1966; Cremonese et al. 2002). The bolometric Bond albedo is a product of the bolometric geometric albedo $(p)$ and the bolometric phase integral $(q)$. In principle, both of these components represent integrals of wavelength-dependent quantities that must be measured and weighted by the solar flux spectrum (Hansen 1977), but they are often approximated as being wavelength independent. Measurements of $p$ for Juno at optical wavelengths $\left(p_{V}\right)$ range from $\approx 0.13$ (Hansen 1977; Brown et al. 1982) to 0.15 (Morrison 1977; Zellner et al. 1977), while a value of $\approx 0.22$ has been found at midinfrared wavelengths (Tedesco et al. 2004; Ryan \& Woodward 2010). The observed variation in optical brightness versus phase angle yields a slope parameter $G=0.17 \pm 0.03$ (Lagerkvist et al. 1992), which in turn yields $q_{V}=0.41 \pm 0.02$ from the relation of Bowell et al. (1989). Combining $q_{V}$ with the more recent measurements of $p_{\mathrm{IR}}$, we will proceed with $A=0.09$, which matches the result others have obtained by using the $p_{V}$ values along with $q_{V}=0.6$, appropriate for the moon. In any case, the dependence of $T_{\text {eq }}$ on $A$ is quite mild. Assuming $\epsilon_{\nu}=1$, the equilibrium model prediction for $T_{B}$ for our Juno observations ( $r=2.072 \mathrm{AU})$ is then $188-223 \mathrm{~K}$, depending on $f$. Juno is a fast rotator, but its polar axis was pointed significantly in line with the Sun during the ALMA observations (Figure 1). Therefore, an $f$ value in the lower half of the range is likely to be appropriate, which is consistent with our measured median $T_{\mathrm{B}}^{\mathrm{obs}}(233 \mathrm{GHz})$ of $197 \pm 15 \mathrm{~K}$. A previous single-dish estimate of $T_{B}^{\mathrm{obs}}(345 \mathrm{GHz})$ was $146 \pm 48 \mathrm{~K}$ at $r=3.132 \mathrm{AU}$ (Chamberlain et al. 2009), which scales to $180 \pm 59 \mathrm{~K}$ at our smaller value of $r$.

Moving beyond the equilibrium model, we next consider the Standard Thermal Model for asteroids (STM; Lebofsky \& Spencer 1989). Because ALMA resolves the surface of Juno, we can compare the observed peak brightness temperature with the expected subsolar surface temperature $\left(T_{\mathrm{ss}}\right)$ in the STM. For Juno's values of $A$ and $r$, and its beaming factor of $\eta=0.76$ (Spencer \& Lebofsky 1989), the expected $T_{\mathrm{ss}}$ is $286 \mathrm{~K}$. This value is significantly higher than the peak $T_{B}$ of $222 \pm 13 \mathrm{~K}$ observed by ALMA, in contrast to previous mid-infrared measurements of $T_{\mathrm{ss}}$ on Juno which are consistent with the 


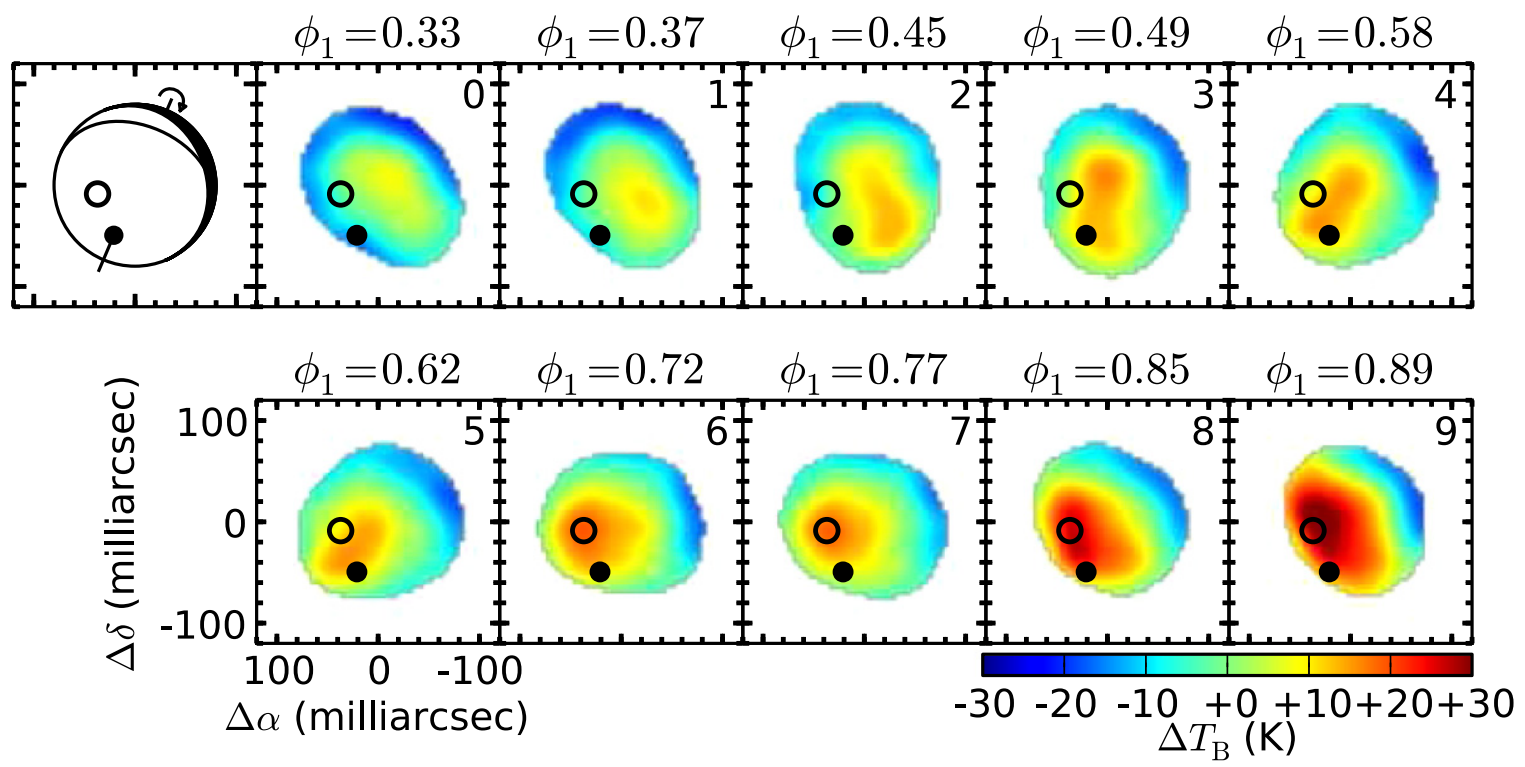

Figure 3. Residual images of Planck brightness temperature $\left(T_{B}\right)$ in equatorial offset coordinates created by removing a model image of uniform brightness from the images in Figure 2. The open circle indicates the location of the subsolar point. The color scale range is $\pm 30 \mathrm{~K}$ with respect to the median values of $T_{B}$ in column 8 of Table 2. The south pole drawn is the same as in Figure 1, and the sense of rotation is clockwise. The different position angle here is due to the combination of the coordinate system, the foreshortening effect of the heavily inclined pole, and the significant heliocentric latitude.

STM prediction (Lim et al. 2005). If interpreted in the context of a model where all of the emission arises from the surface, such a discrepancy could be interpreted as an "effective" $\epsilon_{\nu} \sim 0.8$ at $1.3 \mathrm{~mm}$. Effective emissivity is a quantity that can encompass many effects besides the physical emissivity of the material, including sub-surface sounding of deeper colder layers (Redman et al. 1998; Fornasier et al. 2013). Indeed, in terms of a physical model with temperature gradients in the sub-surface material of up to $50 \mathrm{~K} \mathrm{~mm}^{-1}$, such a large temperature discrepancy is expected to be seen in millimeter wavelength emission, which arises from material at a range of depths, even when the bulk material has $\epsilon_{\nu} \approx 1$ (see, e.g., Keihm et al. 2013).

To interpret the enhanced brightness temperature of the subsolar point in most of the Juno images, we consider heat conduction in the regolith following the equations in Lagerros (1996). The thermal inertia $(\Gamma)$ is a function of the surface material density $(\rho)$, thermal conductivity $(\kappa)$, and specific heat capacity $\left(c_{p}\right)$ of the soil, while the thermal skin depth $\left(l_{s}\right)$ also depends on the angular rotation rate $(\omega)$,

$$
\begin{gathered}
\Gamma=\sqrt{\kappa \rho c_{p}} \\
l_{s}=\sqrt{\frac{\kappa}{\rho c_{p} \omega}}=\frac{\kappa}{\Gamma \sqrt{\omega}} .
\end{gathered}
$$

Using the surface density of Vesta derived from its radar albedo (1.75 $\mathrm{g} \mathrm{cm}^{-3}$; Chamberlain et al. 2009), along with values for $\kappa$ and $c_{p}$ in the porous lunar surface layer $\left(2 \times 10^{-5} \mathrm{~W} \mathrm{~cm}^{-1} \mathrm{~K}^{-1}\right.$ and $0.6 \mathrm{~J} \mathrm{~g}^{-1} \mathrm{~K}^{-1}$, respectively; Keihm 1984), yields $\Gamma=46 \mathrm{~J} \mathrm{~m}^{-2} \mathrm{~s}^{-0.5} \mathrm{~K}^{-1}$ and $l_{s}=2.8 \mathrm{~mm}$. Since $l_{s}$ is only 2.2 wavelengths, we can expect the observed continuum emission to arise from a mix of solar-heated surface material and deeper unheated material. Thus, the correlation of Juno's brightness temperature with the subsolar point is not surprising. However, the fact that the brightest point moves from the subsolar afternoon area in the first half of the images to near coincidence with the subsolar point in the latter half of the images suggests a change in the soil properties with longitude. For example, in the case that $\kappa$ is constant with depth and longitude, $l_{s}$ would scale inversely with $\Gamma$ due to changes in either $\rho$ or $c_{p}$. Thus, if soil with relatively lower values of $\rho$ or $c_{p}$ exists at these longitudes $\left(\phi_{1} \sim 0.7-0.9\right)$, it could have a lower thermal inertia, deeper skin depth, and consequently a greater proportion of the millimeter emission arising from heated material. A lower inertia across most of this side of Juno might also explain the lower median $T_{B}$ observed, as cooling would proceed more rapidly as the angle from the subsolar point increases. In any case, variations in thermal inertia across the surface of an asteroid are quite plausible, particularly in light of the detailed variations on Vesta reported from the Visual and Infrared mapping spectrometer on the Dawn spacecraft (Capria et al. 2014).

Recently, two asteroids have been measured at 0.53 and $1.6 \mathrm{~mm}$ by the Microwave Instrument for the Rosetta Orbiter (MIRO) during close encounters by the European Space Agency Rosetta spacecraft: the small $(\sim 6 \mathrm{~km})$ object Šteins (Gulkis et al. 2010) and the larger $(\sim 100 \mathrm{~km})$ object Lutetia (Gulkis et al. 2012). In contrast to Šteins, which has a high, rocklike inertia $\left(\Gamma=450-850 \mathrm{~J} \mathrm{~m}^{-2} \mathrm{~s}^{-0.5} \mathrm{~K}^{-1}\right)$ and $\epsilon_{\nu}=0.85-0.9$, Lutetia exhibits a very low thermal inertia $\left(\Gamma=20 \mathrm{~J} \mathrm{~m}^{-2} \mathrm{~s}^{-0.5} \mathrm{~K}^{-1}\right)$ in the upper $1-3 \mathrm{~cm}$ much like the fine dust of the lunar regolith, with an emissivity consistent with reflection from a surface with dielectric constant of 2.3 $\left(\epsilon_{\nu}=0.958\right)$. These latter properties may be similar to what ALMA has seen on Juno, particularly in the latter half of the images. This result is perhaps not surprising in the context of the subsequent thermophysical modeling of Keihm et al. (2013), which finds that low thermal inertias and $\epsilon_{\nu} \sim 1$ can fit the infrared to centimeter spectral energy distributions of the asteroids Ceres, Vesta, Pallas, and Hygiea.

The possibility of a recent impact on Juno was raised by the detection of a region of reduced $934 \mathrm{~nm}$ brightness in $\mathrm{AO}$ 
images correlated with a spatial "bite" feature on the limb (Baliunas et al. 2003). Unfortunately, the north/south orientation of the Baliunas et al. (2003) images is not specified. However, judging from the DAMIT model images at that epoch, the AO images would appear to be oriented with south up because in this case, a depression in the model images would map closely to the proposed crater near the limb of the fifth AO image. If so, then the crater is located near the north pole. As shown in Figure 1, the ALMA viewing angle of Juno differs by $114^{\circ}$ from the Baliunas et al. (2003) AO images such that the north pole is not visible. On the other hand, if the AO image is oriented with north up, as it is in similarly acquired images of Vesta by a subset of these authors (Shelton et al. 1997), then the crater would lie between the equator and the south pole, placing it at a latitude that crosses near the center of the ALMA view. The rotational phase of the fifth AO image $\left(\phi_{2}=0.29\right)$ is close to that of ALMA image 3, thus a feature on the limb in the AO image would cross the sub-Earth point in the ALMA image $114^{\circ}+90^{\circ}=204^{\circ}$ later (i.e., at $\left.\phi_{2}=0.86\right)$. This phase corresponds to ALMA images 8 and 9 . Those images do show the highest temperature contrast with respect to the subsolar point, which could be consistent with a lower thermal inertia in the excavated material surrounding the crater. Clearly, future ALMA observations of complete rotations of Juno, preferably at multiple phases and wavelengths, will be necessary to explore this phenomenon further and develop an accurate, full-surface thermophysical model.

\section{CONCLUSIONS}

Our ALMA long-baseline observations of Juno provide the first ground-based images that significantly resolve the surface of an asteroid at millimeter wavelengths. They provide an independent set of size and shape measurements that confirm our current knowledge expressed by the DAMIT and triaxial ellipsoid models. Future ALMA observations of main belt asteroids, including both spatially unresolved photometric light curves (e.g., Moullet et al. 2010) and resolved images, can be used to test and refine the existing three-dimensional models. We note that ALMA can potentially achieve significantly higher physical resolution on Juno than these initial observations offer. For example, a factor of three improvement (to $20 \mathrm{~km}$ resolution) would be possible by observing in the $345 \mathrm{GHz}$ band with a similar antenna configuration at a future favorable opposition (e.g., 2018 November 16, $\Delta=1.04 \mathrm{AU}$ ). These observations would match the resolution of MIRO's $1.6 \mathrm{~mm}$ channel during Rosetta's flyby of Lutetia. At these scales, measurements of the brightness temperature will provide new information about the surfaces of these bodies. To develop accurate thermophysical models, it will be important to observe them at multiple (sub)millimeter wavelengths where a drop in emissivity to values of $\sim 0.6-0.8$ has been reported (e.g., Müller and Lagerros 1998), particularly for rockier bodies (e.g., Gulkis et al. 2010). ALMA can also potentially measure the mutual orbit of smaller binary asteroids, providing important information on the mass and density of such objects (e.g., Carry et al. 2015). Finally, the ability of ALMA to deliver very accurate astrometry will enable better long-term modeling of asteroid orbits, leading to improved predictions (Busch 2009).

This paper makes use of the following ALMA data set: ADS/JAO.ALMA\#2011.0.00013.SV. ALMA is a partnership of ESO (representing its member states), NSF (USA) and NINS (Japan), together with NRC (Canada), NSC and ASIAA (Taiwan), and KASI (Republic of Korea), in cooperation with the Republic of Chile. The Joint ALMA Observatory is operated by ESO, AUI/NRAO and NAOJ. The National Radio Astronomy Observatory is a facility of the National Science Foundation operated under cooperative agreement by Associated Universities, Inc. This research has made use of NASA's Astrophysics Data System. We thank Thomas Müller, Mark Gurwell, Bryan Butler, Rafael Hiriart, Ralph Marson, Dirk Petry, and Vivek Dhawan for useful discussions.

$$
\text { Facilities: ALMA. }
$$

\section{REFERENCES}

ALMA Partnership, Fomalont, E., Vlahakis, C., et al. 2015, ApJL, 808, L1 (ALMA I)

Altenhoff, W. J., Johnston, K. J., Stumpff, P., \& Webster, W. J. 1994, A\&A, 287, 641

Baier, G., \& Weigelt, G. 1983, A\&A, 121, 137

Baliunas, S., Donahue, R., Rampino, M. R., et al. 2003, Icar, 163, 135

Barnard, E. E. 1895, MNRAS, 56, 55

Barnard, E. E. 1900, MNRAS, 61, 68

Birch, P. V., \& Taylor, R. C. 1989, A\&AS, 81, 409

Bowell, E., Hapke, B., Domingue, D., et al. 1989, in Asteroids II, ed. R. Binzel (Tucson, AZ: Univ. Arizona Press), 524

Brown, R. H., Morrison, D., Telesco, C. M., \& Brunk, W. E. 1982, Icar, 52,188

Bus, S. J., \& Binzel, R. P. 2002, Icar, 158, 106

Busch, M. W. 2009, Icar, 200, 347

Campbell, M. J., \& Ulrichs, J. 1969, JGR, 74, 5867

Capria, M. T., Tosi, F., de Sanctis, M. C., et al. 2014, GeoRL, 41, 1438

Carry, B., Matter, A., Scheirich, P., et al. 2015, Icar, 248, 516

Chamberlain, M. A., Lovell, A. J., \& Sykes, M. V. 2007, Icar, 192, 448

Chamberlain, M. A., Lovell, A. J., \& Sykes, M. V. 2009, Icar, 202, 487

Chapman, C. R., Morrison, D., \& Zellner, B. 1975, Icar, 25, 104

Cowling, S. A. 1984, MNRAS, 209, 415

Cremonese, G., Marzari, F., Burigana, C., \& Maris, M. 2002, NewA, 7, 483

Degewij, J., Tedesco, E. F., \& Zellner, B. 1979, Icar, 40, 364

Dollfus, A. 1971, in Proc. IAU Colloquium 12, Physical Studies of Minor Planets, ed. T. Gehrels (NASA Special Publication, 267; Washington DC: NASA), 25

Dotto, E., Barucci, M. A., Müller, T. G., Storrs, A. D., \& Tanga, P. 2002, Asteroids III (Tucson, AZ: Univ. Arizona Press)

Dotto, E., de Angelis, G., di Martino, M., et al. 1995, Icar, 117, 313

Dotto, E., Müller, T. G., Barucci, M. A., et al. 2000, A\&A, 358, 1133

Drummond, J., \& Christou, J. 2008, Icar, 197, 480

Durech, J., Kaasalainen, M., Herald, D., et al. 2011, Icar, 214, 652

Durech, J., Sidorin, V., \& Kaasalainen, M. 2010, A\&A, 513, AA46

Fornasier, S., Lellouch, E., Müller, T., et al. 2013, A\&A, 555, AA15

Gaffey, M. J., Burbine, T. H., \& Binzel, R. P. 1993a, Metic, 28, 161

Gulkis, S., Keihm, S., Kamp, L., et al. 2010, P\&SS, 58, 1077

Gulkis, S., Keihm, S., Kamp, L., et al. 2012, P\&SS, 66, 31

Hansen, O. L. 1977, Icar, 31, 456

Hezaveh, Y. D., Marrone, D. P., Fassnacht, C. D., et al. 2013, ApJ, 767, 132

Hills, R. E., Kurz, R. J., \& Peck, A. B. 2010, Proc. SPIE, 7733, 773317

Kaasalainen, M., Torppa, J., \& Piironen, J. 2002, Icar, 159, 369

Keihm, S. J. 1984, Icar, 60, 568

Keihm, S., Kamp, L., Gulkis, S., et al. 2013, Icar, 226, 1086

Kellermann, K. I. 1966, Icar, 5, 478

Lagerkvist, C.-I., Magnusson, P., Williams, I. P., et al. 1992, Ap\&SS, 94, 43

Lagerros, J. S. V. 1996, A\&A, 310, 1011

Lanyi, G. E., Boboltz, D. A., Charlot, P., et al. 2010, AJ, 139, 1695

Lebofsky, L. A., \& Spencer, J. R. 1989, in Asteroids II, ed. R. Binzel (Tucson, AZ: Univ. Arizona Press), 128

Lim, L. F., McConnochie, T. H., Bell, J. F., \& Hayward, T. L. 2005, Icar, 173,385

Lovell, A. J. 2008, Ap\&SS, 313, 191

Magnusson, P. 1986, Icar, 68, 1

Magri, C., Nolan, M. C., Ostro, S. J., \& Giorgini, J. D. 2007, Icar, 186, 126 Marciniak, A., Bartczak, P., Santana-Ros, T., et al. 2012, A\&A, 545, AA131 Millis, R. L., Wasserman, L. H., Bowell, E., et al. 1981, AJ, 86, 306

Morrison, D. 1977, Icar, 31, 185 
Moullet, A., Gurwell, M., \& Carry, B. 2010, A\&A, 516, L10

Müller, T. G., \& Lagerros, J. S. V. 1998, A\&A, 338, 340

Rau, U., \& Cornwell, T. J. 2011, A\&A, 532, AA71

Redman, R. O., Feldman, P. A., \& Matthews, H. E. 1998, AJ, 116, 1478

Ryan, E. L., \& Woodward, C. E. 2010, AJ, 140, 933

Schroll, A., Schober, H. J., \& Lagerkvist, C. I. 1981, A\&A, 104, 296

Shelton, J. C., Schneider, T., \& Baliunas, S. L. 1997, Proc. SPIE, 3126, 321

Shinokawa, K., Takahashi, S., Ogawa, K., et al. 2002, MmSAI, 73, 658

Spencer, J. R., Lebofsky, L. A., \& Lebofsky, M. V. 1989, Icar, 78, 337
Takahashi, S., Yoshida, F., Shinokawa, K., Mukai, T., \& Kawabata, K. S. 2009, AJ, 138, 951

Tedesco, E. F., Noah, P. V., Noah, M., \& Price, S. D. 2004, IRAS Minor Planet Survey, IRAS-A-FPA-3-RDR-IMPS-V6.0, http://sbn.psi.edu/pds/resource/ imps.html

van Kempen, T., Kneissl, R., Marcelino, N., et al. 2014, ALMA Memo 599, http://library.nrao.edu/public/memos/alma/memo599.pdf

Viikinkoski, M., Kaasalainen, M., \& Durech, J. 2015, arXiv:1501.05958

Zellner, B., Leake, M., Lebertre, T., Duseaux, M., \& Dollfus, A. 1977, LPSC, 8,1091 